Cite this: J. Mater. Chem. C, 2014, 2, 2583

Received 20th December 2013 Accepted 21st January 2014

DOI: $10.1039 / c 3 t c 32525 c$

www.rsc.org/MaterialsC

\section{Redox- and mechano-chromic response of metallopolymer-based elastomeric colloidal crystal films $\dagger$}

\begin{abstract}
D. Scheid, ${ }^{a}$ C. Lederle, ${ }^{\text {b }}$ S. Vowinkel, ${ }^{a}$ C. G. Schäfer, ${ }^{a}$ B. Stühn ${ }^{b}$ and M. Gallei ${ }^{\star a}$
A novel and convenient route for the preparation of monodisperse ferrocene-containing core-interlayershell particles using emulsion polymerisation protocols is described. These metallopolymer-based particles can be used to produce highly ordered elastomeric opal films with fascinating distinct reflection colours. The present work additionally describes the combined addressability of both stimuli, redox-chemistry and mechanical stress. The obtained materials are interesting since application of the melt-shear process followed by crosslinking of the matrix provides access to large-area, mechano-responsive elastomeric opal films featuring an additional redox response. Both, basic synthesis aspects and first steps towards application in the field of stimuli-responsive sensing of respective materials are discussed.
\end{abstract}

\section{Introduction}

Recent efforts in the field of tunable colloidal crystals based on monodisperse organic or inorganic particles featuring a stimuliresponsive core-shell structure or a polymer matrix have led to fascinating stimuli-responsive materials. These materials are promising candidates for, e.g., opto-electronic sensing devices or as actuation systems. ${ }^{1-7}$ By applying external triggers, i.e., by changing the solvent, temperature, ionic strength, light, or by applying an electrical field or mechanical stress, the lattice distances and/or the refractive index of these materials can be tremendously influenced. The response of such materials is accompanied by a remarkably fast change of the optical properties. A great advantage of colloidal crystal structures is their inexpensive and convenient bottom-up preparation resulting in a good optical performance with iridescent reflection colours caused by Bragg diffraction of visible light. ${ }^{8-15}$ For example, a crystalline colloidal array of hydrogel-based photonic crystals has been applied as a sensor showing distinct diffraction colour changes when treated with different transition metal cations or by changing the $\mathrm{pH}$ value. ${ }^{16}$ Very recently, Zhao and co-workers reported the variation of the lattice distances in elastomeric polymeric opals in dependency of an applied voltage. ${ }^{12}$

However, manifold strategies were reported to obtain wellordered and large-scaled 3D arrays based on colloidal spheres.

\footnotetext{
${ }^{a}$ Ernst-Berl-Institute for Chemical Engineering and Macromolecular Chemistry, Technische Universität Darmstadt, Alarich-Weiss-Str. 4, D-64287 Darmstadt, Germany. E-mail:m.gallei@mc.tu-darmstadt.de

${ }^{b}$ Institute of Condensed Matter Physics, Technische Universität Darmstadt, Hochschulstr. 8, D-64289 Darmstadt, Germany

$\dagger$ Electronic supplementary information (ESI) available: Additional synthesis procedures, SEC trace of a statistical PFcMA copolymer, ${ }^{1} \mathrm{H}$ NMR spectrum of the copolymer, DSC thermogram of CIS particles, and TEM images of non-crosslinked particles after oxidation. See DOI: 10.1039/c3tc32525c
}

Drying or spin coating of the particle dispersions are the most common and convenient methods thereof. ${ }^{17-20}$ On the other hand, the so-called melt-flow technique allows the convenient preparation of perfectly ordered polymer and silica particles in elastomeric films. The process for the convenient preparation of large-area self-supporting opal films involves the compression of the monodisperse particles between the plates of a press. ${ }^{21-23}$ The melt-shear technology allows the organization of such coreshell particles consisting of a rigid core and a soft, elastomeric shell into a highly ordered fcc lattice. The soft shell is a basic prerequisite for this process. Elastomeric polymer-based colloidal crystals, also referred to as opal films, show a reversible mechanochromic response while stretching with excellent colour changes due to a modification of the 111 (200) plane spacing. ${ }^{24,25}$ Electrically addressable photonic band gap materials have garnered a great deal of attention in the last few years. Intrinsically conductive polymers such as polyaniline, polypyrrole or polythiophene could be used for this purpose. ${ }^{\mathbf{2 6 - 2 8}}$ Compared to those, reports about stimuli-responsive colloidal structures based on metallopolymers are rather scarce. A major breakthrough in synthesis pathways leading to manifold applications for ferrocene-containing polymers was achieved by Manners' discovery of the ring-opening polymerization of ansaferrocenophanes. ${ }^{\mathbf{2 9 - 3 2}}$ The most prominent example regarding metallopolymers and colloidal crystals was reported by Arsenault et al. The material properties investigated by this group was based on the redox-activity of poly(silaferrocenophane) (PFS) derivatives as matrix materials. ${ }^{\mathbf{1 3}, 33}$ The optical Bragg diffraction of the embedded silica spheres can be varied by oxidizing the ferrocene moieties inducing an increase in the lattice distances.

Due to recent efforts in the synthesis of metal-containing polymers featuring the ferrocene motif in the side-chain, the first steps towards potential applications have been investigated 
in the last few years. ${ }^{34-37}$ Within this contribution, we now expand the field of side-chain ferrocene-containing polymers to novel photonic band gap elastomeric films with combined redox- and mechanochromic properties. The controlled bottomup synthesis of monodisperse redox-responsive particles featuring the ferrocene moieties in the particle shell is accessible using convenient one-pot emulsion polymerization protocols using 2-(methacryloyloxy)ethyl ferrocenecarboxylate (FcMA) as a comonomer with ethyl acrylate (EA). Dynamic light scattering (DLS) is used to prove the tremendous swelling behaviour of the polymer particles after chemical oxidation. Furthermore, the melt-shear technique can be applied to prepare large-scaled redox-responsive opal films which are investigated regarding their combined optical and mechanooptical properties. Our studies provide a convenient method to prepare reversibly and individually addressable well-ordered opal films with fascinating mechanical and redox properties. We expect these functional films to be promising candidates as next generation materials with potential applications in fields of optoelectronic and sensing devices.

\section{Results and discussion}

\section{Free radical (co)polymerisation of FcMA}

With regard to the goal of manufacturing a redox-responsive elastomeric opal film with all its advantages, the use of 2-(methacryloyloxy)ethyl ferrocenecarboxylate (FcMA) for the bottom-up synthesis of core-shell particles obtainable via emulsion polymerisation was investigated. First experiments for the free radical homo- and copolymerisation of the FcMA monomer were carried out to prove the suitability in emulsion polymerisation.

Ferrocene-containing monomers, particularly featuring an electron-rich ferrocene motif, are known for having issues in free radical polymerisation. The radical propagating chain is able to undergo a single electron transfer as evidenced by Georges and Hayes for the vinylferrocene ( $\mathrm{VFc}$ ) monomer. ${ }^{38,39}$ The ability of FcMA to be (co)polymerised with monomers such as ethyl acrylate (EA) in a radical manner is essential for its use in emulsion polymerisation. Studies on polymerisation of the FcMA monomer via ATRP protocols have recently been reported..$^{34,40,41}$

However, more important for the successful manufacture of elastomeric opal films by a convenient melt-shear procedure concerns the resulting glass transition temperature $\left(T_{\mathrm{g}}\right)$ of the shell polymer which builds the matrix in the final opal film. Thus, the free radical copolymerisation of FcMA with ethyl acrylate (EA) is investigated beforehand in this work. Both, homopolymerisation of FcMA and copolymerisation with EA were conducted in solution with AIBN as a thermal initiator. The resulting polymers were characterised by using size-exclusion chromatography (SEC, Fig. S1 $\dagger$ ), DSC (Fig. 1) and NMR (Fig. S2 $\dagger$ ). The experiments succeeded with respect to reasonable molar masses and monomer ratios of FcMA and EA used in the corresponding copolymers (see $\mathrm{ESI}_{\dagger}^{\dagger}$ ). The $T_{\mathrm{g}}$ of the copolymer obtained $\left(\mathrm{PFcMA}_{15 \%}-\mathrm{Co}-\mathrm{PEA}_{\mathbf{8 5} \%}\right)$ and the corresponding homopolymers (PFcMA and PEA) ${ }^{22,34}$ are compared by using

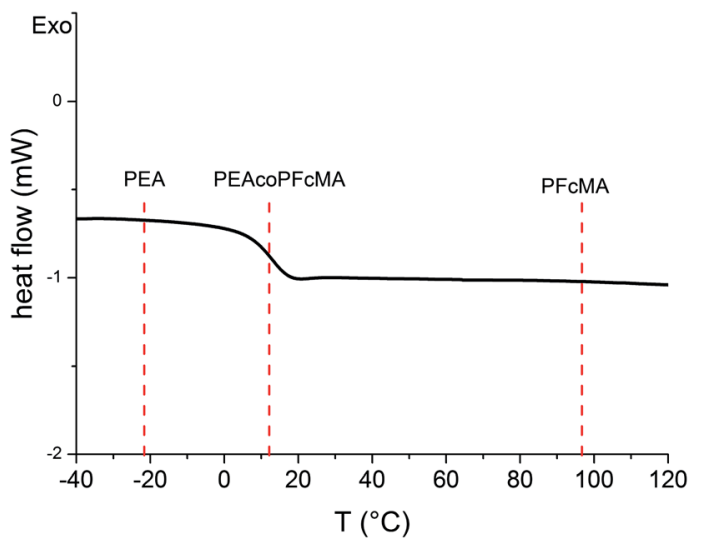

Fig. 1 DSC thermogram of synthesised $P(F C M A-C O-E A)$ and expected $T_{\mathrm{g}}$ values for PEA and PFCMA $\left(M_{\mathrm{w}}=15 \mathrm{kDa}\right)$ homopolymers.

DSC measurements (Fig. 1) proving the successful copolymerization yielding a melt-flow processable ferrocene-containing polymer. Due to the moderate $T_{\mathrm{g}}$ of $11{ }^{\circ} \mathrm{C}$ for the shell polymer, the obtained film will also show elastomeric behaviour after appropriate matrix crosslinking.

\section{Emulsion (co)polymerisation of the FcMA monomer}

Since the monodispersity of core-shell particles is a basic prerequisite for colloidal crystallisation, the introduction of the FcMA monomer in emulsion polymerisation is a crucial step in order to produce a ferrocene-containing elastomeric opal film in the next step. Fig. 2A (top) shows schematically the stepwise bottom-up growth of the core-interlayer-shell (CIS) particles starting from hard PS core particles followed by the formation of a slightly crosslinked interlayer. In the final step, FcMA was copolymerized with EA to generate the soft, redox-responsive shell. Evidence for the desired monodispersity and the attainment of an appropriate particle diameter is given by TEM images (Fig. 2). Additionally, the growth was followed by dynamic light scattering (DLS) after each synthetic step (Fig. 2).

The DSC measurement in Fig. S3† shows a $T_{\mathrm{g}}$ for the synthesized particles above the $T_{\mathrm{g}}$ of PEA, confirming the statistic incorporation of FcMA into the shell polymer. Furthermore, the $T_{\mathrm{g}}$ is still lower than the ambient temperature for the desired film properties. The second $T_{\mathrm{g}}$ above $110^{\circ} \mathrm{C}$ can be assigned to the crosslinked PS cores.

Altogether, we were able to synthesize monodisperse CIS particles with redox responsive FcMA in the shell polymer and the obtained latex matches in all requirements for the manufacture of elastomeric opal films via the melt-shear procedure. Before we turn to the investigation of redox-responsive behaviour of the particles, the attempts for opal film preparation will be described in the next section.

\section{FcMA containing elastomeric opal films}

The next step after the successful synthesis of FcMA-containing CIS particles was fabrication of elastomeric opals via the meltshear technique. Therefore, the FcMA-containing particles were 


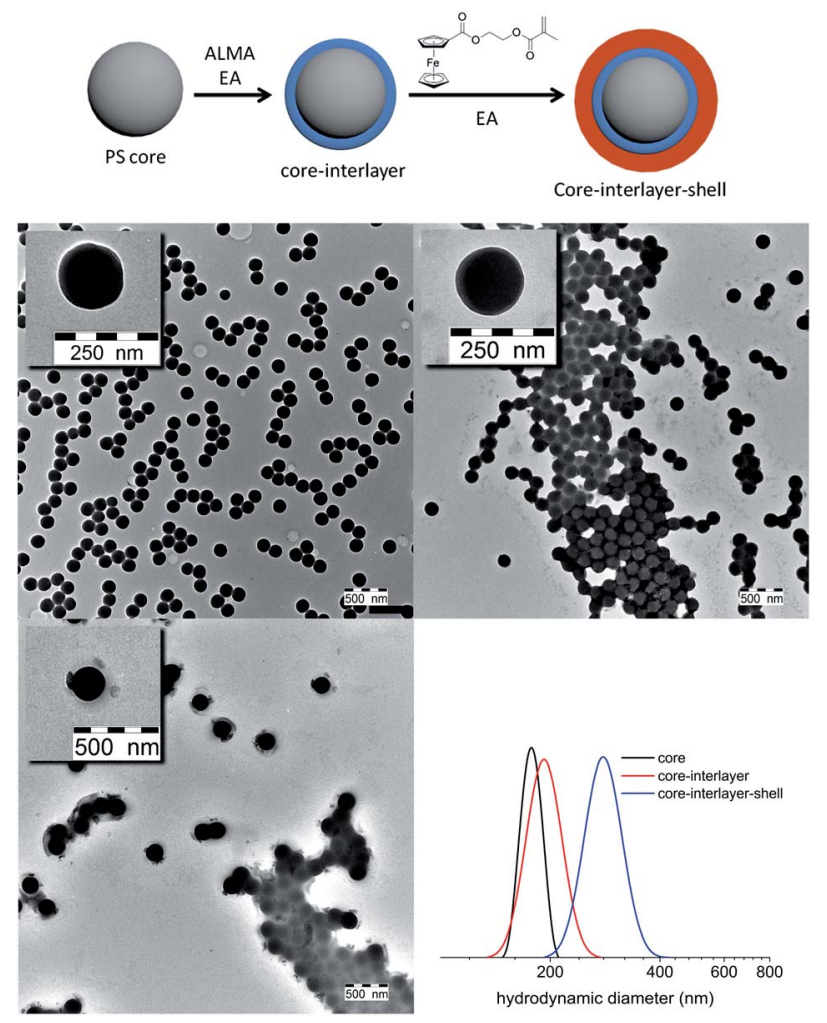

Fig. 2 Scheme of built-up particle architecture (top), TEM images of each stage of synthesis and the stepwise particle growth obtained by using DLS measurements.

precipitated and manufactured into elastomeric opal films in the melt-shear process based on previously described routes..$^{21,22}$ For elastomeric behaviour and, thus, for the mechanochromic response, benzophenone as an UV-active crosslink agent was homogeneously incorporated during the manufacturing process and the final film was irradiated by UV light.

The average particle diameters were chosen to be $176 \pm 5 \mathrm{~nm}$ for the core and $269 \pm 6 \mathrm{~nm}$ for the CIS particles respectively as determined by using DLS measurements (Fig. 2). These particle diameters correspond to a green reflection colour of the prepared films at normal light incidence (angle of light incidence $\theta=90^{\circ}$ ) for the final multi-stimuli responsive material. The size of the redox-responsive CIS particles can be adjusted in the range of 150 to $500 \mathrm{~nm}$. The obtained colours confirmed expectations ensuing from the determined particle size as can be calculated by using Bragg's law. The resulting film disk revealing diameters of about $15 \mathrm{~cm}$ show remarkably brilliant reflection colours which can be switched by changing the angle of view or by stretching the films to a defined strain $\varepsilon$. Photographs of the obtained opal film featuring the expected reflection colours are shown in Fig. 3A and B. In contrast, the non-ordered actual material prior to the applied melt-shearing process revealed a brownish yellow colour.

Additionally, the optical properties of the obtained films were characterised by UV/VIS spectroscopy to confirm the optical similarity of the new material to conventional elastomeric opals. Fig. 3C shows the typical blue shift when

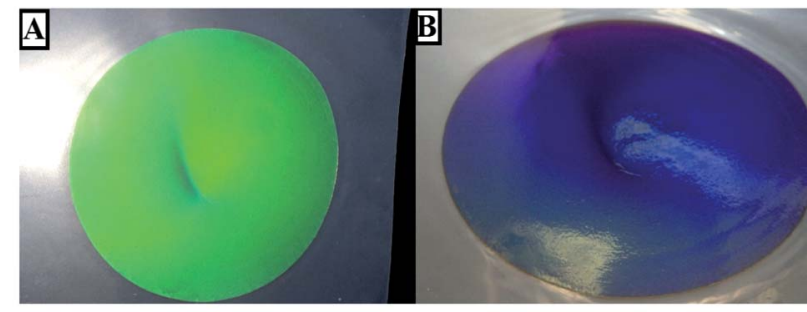

过

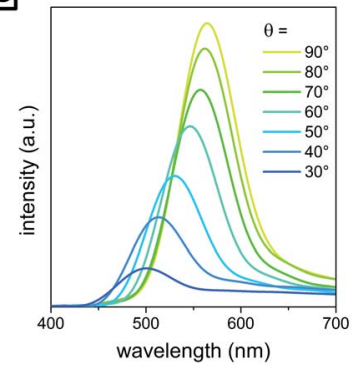

D

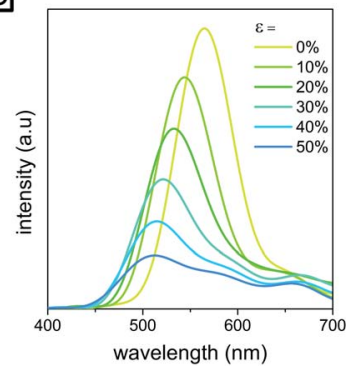

Fig. 3 Photographs of the obtained opal films at different angles of light incidence and the respective angles of view (A) at normal light incidence $\theta=90^{\circ}$, (B): at $\theta=45^{\circ}$, angle dependent UV/VIS reflection measurements $(\mathrm{C})$ and UV/VIS reflection measurements at strains from $\varepsilon=0 \%$ to $\varepsilon=50 \%$ (D).

decreasing the angle of incidence from $90^{\circ}$ to $30^{\circ}$. The blue shift induced by the strain up to $50 \%$ is depicted in Fig. 3D and can be assigned to the decreasing 111 lattice plane spacing of the colloidal crystal structure.

\section{Chemical redox-response of FcMA-containing latex particles}

In the next step, the redox-response of FcMA-containing CIS particles was studied to evaluate the possibility of redoxinduced swelling of the particles inside the elastomeric colloidal crystalline structure. Therefore, the oxidation of CIS particles in aqueous media is supposed to cause significant swelling behaviour as illustrated in Fig. 4 comparable to previous results obtained for PFcMA-brushes immobilized using organic polystyrene particles. ${ }^{34}$

The polymer shell of CIS particles designed for the preparation of elastomeric opal films tended to peel off after oxidation (as evidenced by TEM measurements, Fig. S4†). This phenomenon is caused by the absence of any crosslinking agent in the soft polymer shell. Thus, it is worthy to note that a crosslinking of the polymer shell with allyl methacrylate (ALMA) was sine qua non for oxidation experiments, since only a few

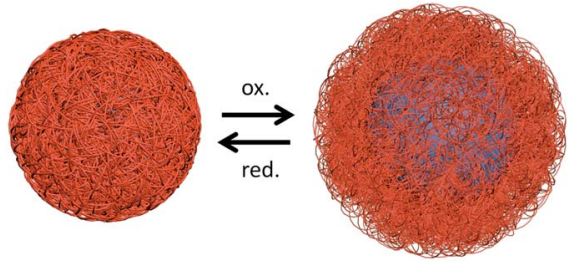

Fig. 4 Chemically induced redox-switching of the CIS particle diameter in an aqueous medium. 
polymer chains were grafted to the interlayer. Concerning these aspects, emulsion polymerisation with a modified mechanism was conducted, resulting in latex particles whereas the shell polymer consists of 82\% EA, 15\% FcMA and 3\% ALMA (experimental details are given in the ESI $\dagger$ ).

Chemical oxidation of the obtained ferrocene-containing latex was carried out by treatment with $\mathrm{FeCl}_{3}$ in water. Collaborative TEM studies revealed the stability of the PFcMA-containing latex after oxidation and subsequent reduction with ascorbic acid (Fig. 5). These results prove the feasibility of the PFcMA-containing particles to be reversibly addressable by the redox stimulus used.

DLS measurements of the particle dispersion before and after oxidation prove the successful redox-induced switching of the ferrocene/ferrocenium moieties (Fig. 6). The particle polydispersity was found to be $9.2 \%$ before oxidation and $15.3 \%$ after, calculated using a cumulant analysis. The correlation time $\tau$ is related to the translational diffusion coefficient $D$ by $D$ $=1 /\left(\tau q^{2}\right)$. The expected linear variation of $1 / \tau$ with $q^{2}$ was well

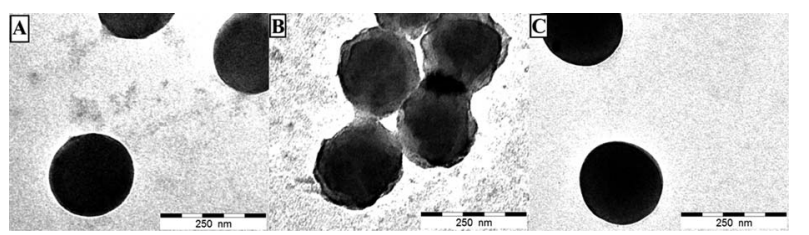

Fig. 5 TEM measurements of the original (A), the oxidised (B) and the reduced $(C)$ particles. The corresponding diluted particle dispersions were drop-cast onto a carbon-coated copper grid.
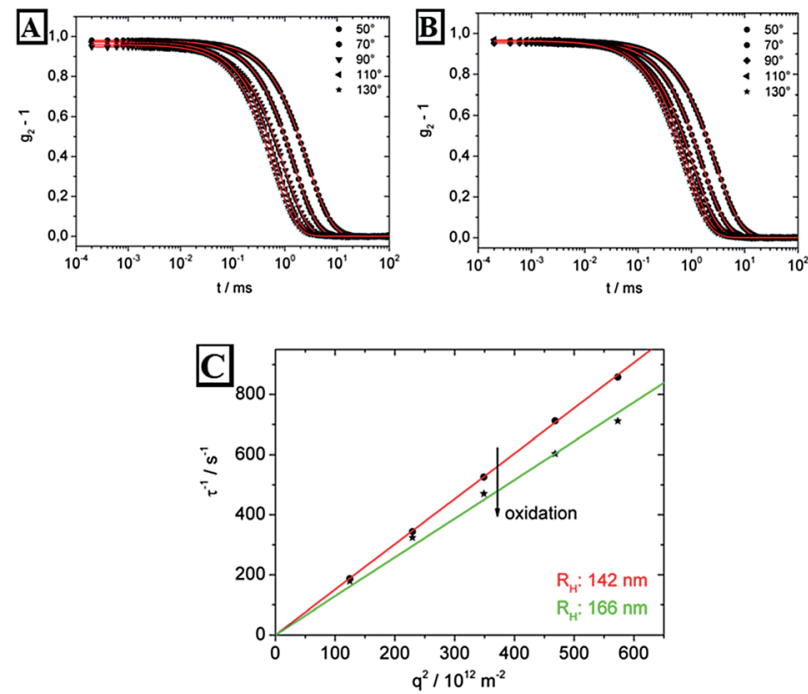

Fig. 6 Intensity autocorrelation functions for the original (A) and oxidised (B) PFCMA-containing particles in water at 20:50-130', measured at $20^{\circ} \mathrm{C}$. The solid lines represent the fit of the data using a cumulant analysis. (C) $q^{2}$ dependence of inverse relaxation times to determine the diffusion coefficient and the resultant hydrodynamic radii for different particles before and after oxidation. Prior to oxidation, the hydrodynamic radius could be determined to be $142 \mathrm{~nm}$ (284 nm diameter) and $166 \mathrm{~nm}$ (332 nm diameter) after oxidation. Errors for the hydrodynamic radii are less than $2 \%$. represented by our data. By using the Stokes-Einstein relation a hydrodynamic radius $R_{\mathrm{h}}=k_{\mathrm{B}} T / 6 \pi \eta D$ could be calculated, with $k_{\mathrm{B}}, T$, and $\eta$ as the Boltzmann constant, the absolute temperature, and the solvent viscosity, respectively. Oxidised PFcMAcontaining particles show a tremendous increase of additional $24 \mathrm{~nm}$ of the hydrodynamic radius $R_{\mathrm{h}}$ suitable for intended swelling-induced optical changes for the novel opal films.

These results unambiguously prove the redox-induced swelling capability of CIS particles in water.

In summary, the redox properties of the CIS particles in water enabled a significant increase of the particle diameter after oxidation due to the oxidation of the ferrocene moieties. These oxidation protocols were applied for switching the opal film properties regarding their polarity and underlying lattice plane spacing accompanied by a significant change of reflection colours as described in the following sections.

\section{Redox response of PFcMA-containing elastomeric opal films}

Treatment of the opal film with $\mathrm{FeCl}_{3}$ in THF caused a more homogeneous invasion of the oxidation agent into the film compared to the first attempts with $\mathrm{FeCl}_{3}$ in water. The original film was capable of swelling in THF but not in water. Hence, oxidation had to be carried out in a good solvent for the particle shell. In the dry state, the film retains its original green reflection colour independent of the presence of an oxidation reagent used (Fig. 7). That means that in the bulk state, the chemically induced swelling was negligible.

Interestingly, the dried opal film revealed a tremendous change of its reflection colour from initial green to red after treatment with water caused by the swelling of the oxidised matrix polymer (Fig. 8).

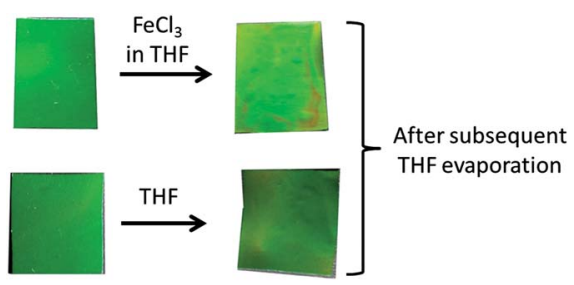

Fig. 7 Photographs of the PFCMA-containing elastomeric opal films before and after chemical oxidation followed by drying. The optical response in the presence of an oxidation reagent was negligible in the dry state. A blank test (treated with THF only) is given for comparison.

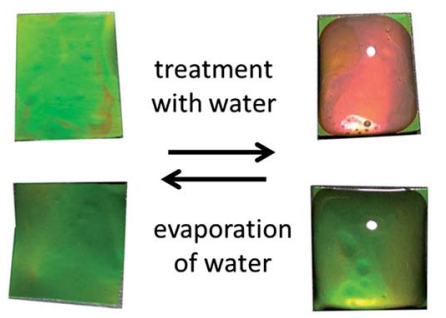

Fig. 8 Oxidised film and blank test treated with water. 
The water induced colour change of the oxidised opal film is fully reversible, once the residual moisture is evaporated. Time dependence and reversibility are determined by UV/VIS reflection measurements. The shift of the Bragg peak dependent on time, and therefore, dependent on the swelling degree is demonstrated in Fig. 9.

The UV/VIS spectra shown in Fig. 9 (top) confirm a swelling induced reflection colour shift of slightly more than $100 \mathrm{~nm}$, which means a change from green to red reflection colour under normal light incidence. It is clearly observable that the initial Bragg peak - corresponding to a green wavelength range - is decreasing immediately in the presence of water. A second signal appears at $\sim 600 \mathrm{~nm}$ and moves up to $690 \mathrm{~nm}$ upon further swelling.

Also, the spectra of the oxidised film prior to swelling and after water evaporation (Fig. 9 bottom) confirm the complete reversibility of the swelling process featuring the original stop band (shifted less than $5 \mathrm{~nm}$ ) after relaxation. The 3D structure persisted due to the crosslinks of the matrix polymer.

Besides the convenient large-scalable opal film manufacture, the mechanical response of the film is an important advantage compared to other colloidal crystal materials. Concerning this aspect, the combination of redox and mechanical responses is of particular importance. Since the elasticity is due to matrix
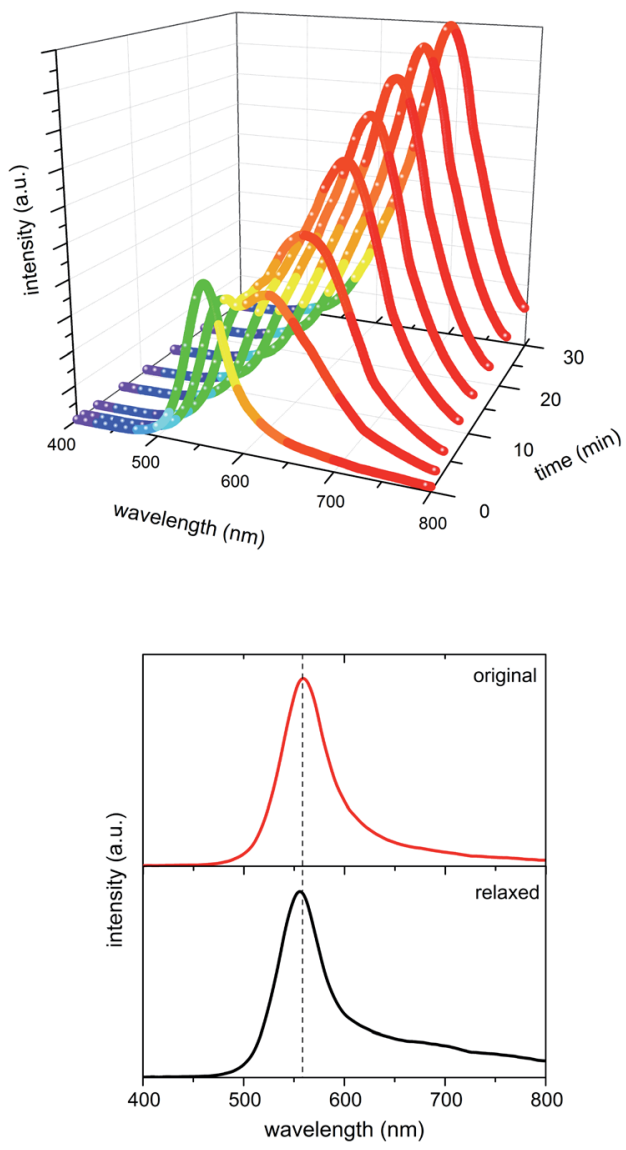

Fig. 9 Time dependent UV/VIS reflection measurements during water swelling of elastomeric opal films (top) and Bragg peaks of a film before swelling and after water evaporation (bottom). polymer crosslinking, the mechanochromic behaviour is still available in the oxidized and the swollen states. The individual addressability of combined stimuli is schematically depicted in Fig. 10.

As shown in Fig. 10, the swelling process as well as the mechanical deformation of the elastomeric opal film leads to a change of lattice distances. This change is responsible for the resulting angle dependent reflection colour.

For the determination of the strain induced colour shift, the ferrocenium-containing film was swollen in water and fixed in a stretch gadget (see Fig. 11, top). The corresponding UV/VIS reflection measurements are shown in Fig. 11.

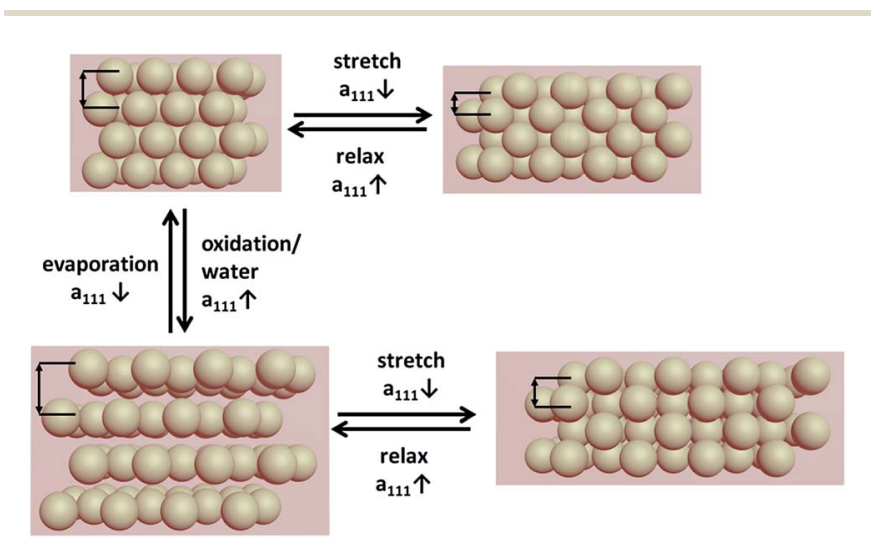

Fig. 10 Change of lattice plane spacing induced by swelling of the opal film after oxidation and strain induced deformation of the crystal lattice.
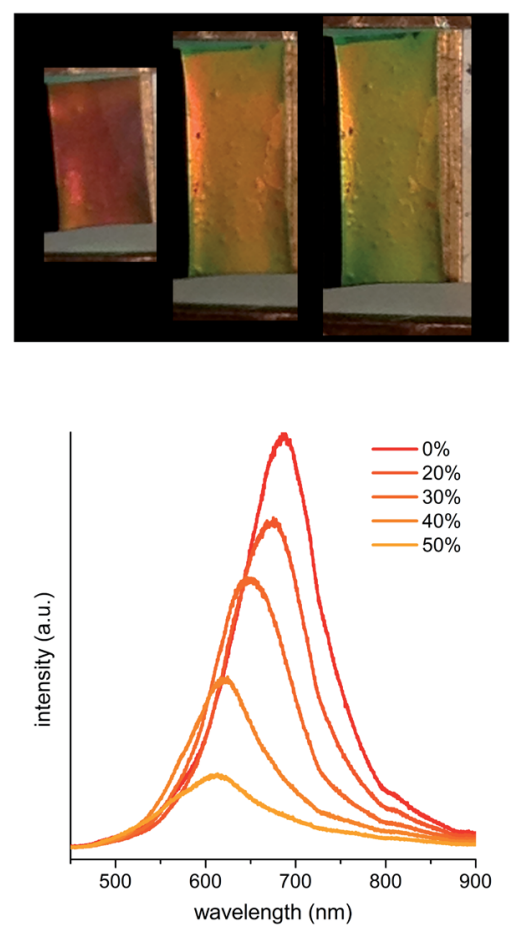

Fig. 11 (Top) Exemplary strain experiments of oxidized metallopolymer-based opal films (UV-crosslinked with 3 wt\% benzophenone) under water. (Bottom) UV/VIS reflection measurements of the oxidised opal film in water dependent on applied strain. 


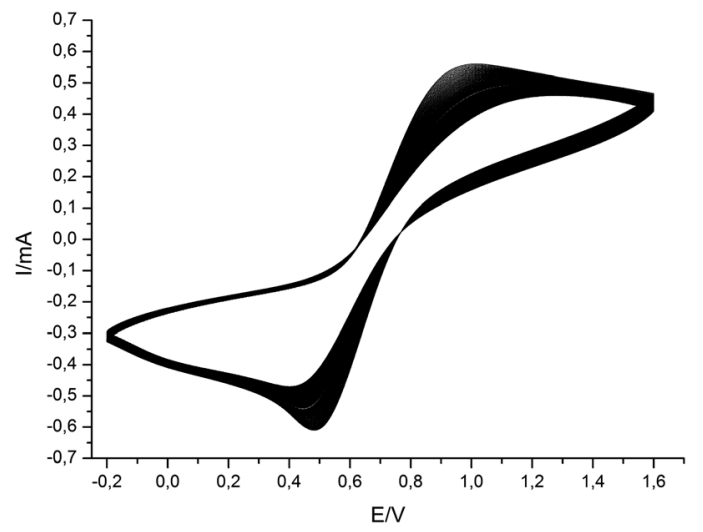

Fig. 12 Cyclic voltammograms of the ferrocene-containing elastomeric opal film fixed on an ITO slide. The applied potential was varied from $-0.2 \mathrm{~V}$ to $+1.6 \mathrm{~V}$ in 80 cycles in acetonitrile and tetrabutylammonium hexafluorophosphate $(0.1 \mathrm{M})$ as the electrolyte.

The UV/VIS reflection spectra of swollen, oxidised films confirm the mechanocromic behaviour of this novel metallopolymer-based photonic crystal material.

\section{Electric response of PFcMA-containing elastomeric opal films}

For the first steps towards future applications of herein investigated redox-responsive elastomeric films in fields of sensing applications and display technologies, the electro-chemical response of the obtained opal films was investigated by cyclic voltammetry experiments. The corresponding cyclic voltammograms are given in Fig. 12. For these experiments the film was fixed on a conductive indium tin oxide (ITO) plate in the presence of tetrabutylammonium hexafluorophosphate as the electrolyte in acetonitrile.

The cyclic voltammograms show the typical redox signals for ferrocene-containing polymers with minima and maxima lying in between 400 and $900 \mathrm{mV}$ as previously reported for the diluted polymers. ${ }^{34}$ The endurance of the manufactured material during 80 iterations confirms the reversible redox responsiveness of ferrocene repetitive units in the opal film matrix. These first experiments unambiguously reveal the suitability for the as-prepared films to be repeatedly addressable by applying a current which gives rise to manifold future applications in the field of soft sensing.

\section{Conclusions}

The ferrocene-containing monomer 2-(methacryloyloxy)ethyl ferrocenecarboxylate (FcMA) was successfully radically polymerised leading to monodisperse metallopolymer-based stimuli-responsive particles featuring a core-interlayer-shell (CIS) architecture by applying convenient emulsion polymerisation protocols. These novel materials were excellent candidates for a feasible preparation of large-scaled elastomeric opal films by the so-called melt-shear technique featuring distinct iridescent reflection colours corresponding to Bragg's law. Essential for the successful film preparation- which was exclusively based on the particle architecture - was the narrow particle size distribution as well as an appropriate glass transition temperature of the CIS-building segments. For this purpose, parameters for copolymerising FcMA and ethyl acrylate (EA) monomers in solution and in emulsion polymerisation were thoroughly investigated and successfully adjusted to the process. The ferrocene-containing repetitive units revealed the intrinsic capability to change the particle polarity after oxidation accompanied by tremendous particle swelling which was investigated by using DLS measurements before and after chemical oxidation. The finally prepared ferrocene-containing elastomeric opal films were studied regarding their mechanochromic response showing brilliant and iridescent reflection colours corresponding to Bragg's law. The excellent redoxresponsive behaviour for the particles in dispersion and for the elastomeric opal film was evidenced by DLS measurements and UV/VIS reflection spectroscopy, respectively. Moreover, we were able to confirm the typical mechanochromic behaviour of the obtained opal films in the dry state prior to and after oxidation. For this purpose, different strains for a water-swollen oxidised ferrocene-containing film were applied showing the individual addressability of both stimuli used, redox chemistry and mechanical stress. The fully reversible ferrocene reduction/ oxidation cycles inside the prepared opal films were studied by cyclic voltammetry for at least 80 cycles. We expect herein prepared novel functional materials as interesting candidates for manifold sensing applications and future display technologies based on stimuli-responsive polymeric materials.

\section{Materials and methods}

\section{Reagents}

Styrene, EA, and butanediol diacrylate (BDDA) were obtained from BASF SE; ALMA and MMA from Evonik Rohm GmbH; and Dowfax 2A1 from Dow Chemicals. FcMA was synthesised according to the literature. ${ }^{30}$ All other chemicals were purchased from VWR and Sigma Aldrich and used as received, if not otherwise stated. Prior to use in emulsion polymerisation, the stabilisers were removed from the monomers. For this purpose, EA and MMA were extracted with $1 \mathrm{M}$ sodium hydroxide solution, washed with water until the solution was neutral, and then dried over sodium sulfate. $\mathrm{S}$ was distilled under reduced pressure. ALMA and BDDA were destabilised using an ion exchanger (De-Hibit 200, PolySciences Europe GmbH).

\section{Instrumentation}

NMR spectra were recorded on a Bruker DRX 500 spectrometer working at $500 \mathrm{MHz}\left({ }^{1} \mathrm{H} \mathrm{NMR}\right)$. NMR chemical shifts are referenced relative to tetramethylsilane. Standard SEC was performed with THF as the mobile phase (flow rate $1 \mathrm{~mL} \mathrm{~min}^{-1}$ ) on a SDV column set from PSS (SDV 1000, SDV 100000, SDV $1000000)$ at $30{ }^{\circ} \mathrm{C}$. Calibration was carried out using PS standards (from Polymer Standard Service, Mainz). TEM experiments were carried out on a Zeiss EM 10 electron microscope operating at $60 \mathrm{kV}$. All shown images were recorded with a slowscan CCD camera obtained from TRS (Tröndle) in bright field mode. Camera control was computer-aided using the ImageSP 
software from TRS. CV measurements were carried out on a multi-potentiostat VMP2 (Princeton Applied Research) with a custom-made cell in dry acetonitrile with tetrabutylammonium hexafluorophosphate (TBAHFP) as the electrolyte (0.1 M) under a nitrogen atmosphere. $\mathrm{The} \mathrm{Ag} / \mathrm{AgCl}$ reference electrode and $\mathrm{Pt}$ counter electrodes were chosen and a scan rate of $100 \mathrm{mV} \mathrm{s}^{-1}$ in a range of $-0.2 \mathrm{~V}$ to $1.6 \mathrm{~V}$ was applied. Dynamic light scattering (DLS) experiments were carried out with a set-up based on a HeNe laser $(\lambda=632.8 \mathrm{~nm})$ as the light source. Polarization of the primary beam is defined using a Glan-Thomson prism. The scattered beam polarization was analyzed in the verticalvertical geometry. The scattered intensity was detected with an optical fiber coupled to two avalanche photodiodes. The intensity autocorrelation functions, calculated with ALV 5000 software for both photodiodes, were measured in steps of $20^{\circ}$, usually in the angle range between $2 \theta=50^{\circ}$ to $130^{\circ}$. The measurement time for each angle was $5 \mathrm{~min}$. All measurements were performed at $20^{\circ} \mathrm{C}$ using cylindrical cuvettes (Hellma) for the samples in a temperature controlled index matching bath. DSC measurements were recorded with a Mettler Toledo DSC1 from $-50{ }^{\circ} \mathrm{C}$ to $100{ }^{\circ} \mathrm{C}$ with a heating rate of $10 \mathrm{~K} \mathrm{~min}^{-1}$. Additional DLS measurements of the particles were performed on a Nanophox photon cross-correlation spectrometer (Sympatec). The experiments on diluted dispersions of the particles after each step of the synthesis were carried out at an angle of $90^{\circ}$ at $20^{\circ} \mathrm{C}$. Reflection spectra were recorded using a vis-NIR fiber spectrophotometer (USB 4000, Ocean Optics). For reflection measurements a deuterium/tungsten halogen lamp (HL2000, Ocean Optics) was used. Reflection measurements as a function of strain $\varepsilon$ respectively in air and water were carried out at normal light incidence. Therefore, a $1 \times 2 \mathrm{~cm}$ strip of the films was uniformly stretched with a custom-made micrometrecontrolled sample holder, while the optical properties were measured simultaneously. Angle-dependent reflection measurements were carried out using a custom-built goniometer setup. Swelling of the oxidized opal film was tracked after setting a drop of water on the top of the opal films. For the timedependent measurement of the swelling process at room temperature $\left(22^{\circ} \mathrm{C}\right)$ spectra were recorded at intervals of $1 \mathrm{~min}$. Reversible switching of the stop band was followed by recording reflection spectra after each cycle.

\section{Synthesis of CIS particles}

2-(Methacryloyloxy)-ethyl ferrocenecarboxylate (FcMA) containing CIS particles were synthesised by starved feed emulsion polymerization. First, the PS cores were produced in a $1 \mathrm{~L}$ vessel equipped with a stirrer, a reflux condenser and nitrogen feeding at $75{ }^{\circ} \mathrm{C}$ as follows. An ice cooled mixture of $320 \mathrm{mg}$ sodium dodecyl sulfate (SDS), $280 \mathrm{~g}$ deionised water, $3.6 \mathrm{~g}$ styrene and $0.4 \mathrm{~g}$ BDDA was filled in the vessel, immediately followed by the addition of $36 \mathrm{mg}$ sodium disulfite (NaDS), $520 \mathrm{mg}$ sodium persulfate (NaPS) and $36 \mathrm{mg}$ NaDS in this order (each component is dissolved in $5 \mathrm{~mL}$ water). Then, $10 \mathrm{~min}$ after clouding, an emulsion of $230 \mathrm{mg}$ SDS, $400 \mathrm{mg}$ potassium hydroxide $(\mathrm{KOH})$, $220 \mathrm{mg}$ Dowfax 2A1, $90 \mathrm{~g}$ water, $70 \mathrm{~g}$ styrene and $7 \mathrm{~g}$ BDDA continuously added with a rate of $1 \mathrm{~mL} \mathrm{~min}{ }^{-1}$. After complete addition of monomer emulsion, the reaction was finished by holding the temperature for an additional hour. The CIS particle synthesis was conducted in a $250 \mathrm{~mL}$ vessel with the same equipment at $75{ }^{\circ} \mathrm{C} .113 .75 \mathrm{~g}$ of occurred PS latex was filled in the vessel. After adding $25 \mathrm{mg}$ NaDS, $100 \mathrm{mg}$ NaPS and $25 \mathrm{mg}$ NaDS (dissolved each in $3 \mathrm{~mL}$ water), the monomer emulsion containing $13 \mathrm{mg}$ SDS, $53 \mathrm{mg}$ Dowfax 2A1, $8 \mathrm{~g}$ deionised water, $5.55 \mathrm{~g}$ EA and $0.75 \mathrm{~g}$ ALMA was added with a flow rate of $0.25 \mathrm{~mL} \mathrm{~min}{ }^{-1}$, followed by the addition of $100 \mathrm{mg}$ SDS, $100 \mathrm{mg} \mathrm{KOH}, 300 \mathrm{mg}$ Dowfax 2A1, $40 \mathrm{mg}$ water, $30.17 \mathrm{~g}$ EA and $5.33 \mathrm{~g}$ FcMA under similar conditions. After complete addition of monomer emulsion, the reaction was finished by maintaining the temperature for an additional hour.

\section{Opal film preparation}

For the preparation of elastomeric opal films, the obtained CIS particles were precipitated in methanol, containing a small amount of saturated sodium chloride solution. The precipitate was filtered, water washed and dried under reduced pressure. The resulting elastomeric mass was compacted and mixed with benzophenone (3 $\mathrm{wt} \%$ ) in a microextruder (microl, DSM Research) at $130{ }^{\circ} \mathrm{C}$. For enhanced colour contrast, $0.15-0.5 \%$ of carbon nanotubes (BAYTUBES C 150 P, Bayer Material Science) were additionally incorporated. A $3 \mathrm{~g}$ portion of the rubbery polymer was covered with a PET film and heated to $130{ }^{\circ} \mathrm{C}$ between the plates of a Collin 300E laboratory press. Melt flow was induced by applying 150 bar for $3 \mathrm{~min}$, resulting in an elastomeric opal film of about $15 \mathrm{~cm}$ in diameter. Finally, the film was irradiated with an industrial-type mercury lamp (UV Cube 2000, Dr Hoenle) with an output power of $1000 \mathrm{~W}$. The PET-covered films were treated at a distance of $4 \mathrm{~cm}$ for $2 \mathrm{~min}$ each side.

\section{Acknowledgements}

The authors thank the Landesoffensive zur Entwicklung Wissenschaftlich-ökonomischer Exzellenz (LOEWE Soft Control) for on-going financial support of this work. Our thanks also go out to Johannes Elbert and Margarita Balyschewa for collaboration in synthesis work, as well as Sabrina Mehlhase, Marion Trautmann and Matthias Wittemann for analytical support (DSC, SEC).

\section{Notes and references}

1 J. Ge and Y. Yin, Angew. Chem., Int. Ed., 2011, 50, 1492-1522. 2 Y. Xia, B. Gates, Y. Yin and Y. Lu, Adv. Mater., 2000, 12, 693713.

3 A. P. Hynninen, J. H. Thijssen, E. C. Vermolen, M. Dijkstra and A. van Blaaderen, Nat. Mater., 2007, 6, 202-205.

4 M. Maldovan and E. L. Thomas, Appl. Phys. Lett., 2006, 88, 251907.

5 R. De La Rue, Nat. Mater., 2003, 2, 74-76.

6 L. Gonzalez-Urbina, K. Baert, B. Kolaric, J. Perez-Moreno and K. Clays, Chem. Rev., 2012, 112, 2268-2285. 
7 Y. Zhang, J. Wang, Y. Huang, Y. Song and L. Jiang, J. Mater. Chem., 2011, 21, 14113.

8 F. Marlow, Muldarisnur, P. Sharifi, R. Brinkmann and C. Mendive, Angew. Chem., Int. Ed., 2009, 48, 6212-6233.

9 A. Stein, F. Li and N. R. Denny, Chem. Mater., 2008, 20, 649666.

10 J. Zhang, Y. Li, X. Zhang and B. Yang, Adv. Mater., 2010, 22, 4249-4269.

11 S. Y. Lee, L. Gradon, S. Janeczko, F. Iskandar and K. Okuyama, ACS Nano, 2010, 4, 4717-4724.

12 Q. Zhao, A. Haines, D. Snoswell, C. Keplinger, R. Kaltseis, S. Bauer, I. Graz, R. Denk, P. Spahn, G. Hellmann and J. J. Baumberg, Appl. Phys. Lett., 2012, 100, 101902-101904.

13 A. C. Arsenault, D. P. Puzzo, I. Manners and G. A. Ozin, Nat. Photonics, 2007, 1, 468-472.

14 A. Vlad, A. Frölich, T. Zebrowski, C. A. Dutu, K. Busch, S. Melinte, M. Wegener and I. Huynen, Adv. Funct. Mater., 2013, 23, 1164-1171.

15 G. von Freymann, V. Kitaev, B. V. Lotsch and G. A. Ozin, Chem. Soc. Rev., 2013, 42, 2528-2554.

16 H. Jiang, Y. Zhu, C. Chen, J. Shen, H. Bao, L. Peng, X. Yang and C. Li, New J. Chem., 2012, 36, 1051-1056.

17 B. Griesebock, M. Egen and R. Zentel, Chem. Mater., 2002, 14, 4023-4025.

18 H.-L. Li and F. Marlow, Chem. Mater., 2006, 18, 1803-1810.

19 P. Jiang and M. J. McFarland, J. Am. Chem. Soc., 2004, 126, 13778-13786.

20 A. Mihi, M. Ocaña and H. Míguez, Adv. Mater., 2006, 18, 2244-2249.

21 C. G. Schäfer, M. Gallei, J. T. Zahn, J. Engelhardt, G. P. Hellmann and M. Rehahn, Chem. Mater., 2013, 25, 2309-2318.

22 C. G. Schäfer, D. A. Smolin, G. P. Hellmann and M. Gallei, Langmuir, 2013, 29, 11275-11283.

23 C. G. Schäfer, B. Viel, G. P. Hellmann, M. Rehahn and M. Gallei, ACS Appl. Mater. Interfaces, 2013, 5, 1062310632 .
24 B. Viel, T. Ruhl and G. P. Hellmann, Chem. Mater., 2007, 19, 5673-5679.

25 H. Fudouzi and T. Sawada, Langmuir, 2006, 22, 1365-1368.

26 G. H. Shim and S. H. Foulger, Photonics and Nanostructures, 2012, 10, 440-446.

27 Y. Imai, C. E. Finlayson, P. Goldberg-Oppenheimer, Q. Zhao, P. Spahn, D. R. E. Snoswell, A. I. Haines, G. P. Hellmann and J. J. Baumberg, Soft Matter, 2012, 8, 6280.

28 M. Heim, S. Reculusa, S. Ravaine and A. Kuhn, Adv. Funct. Mater., 2012, 22, 538-545.

29 G. R. Whittell and I. Manners, Adv. Mater., 2007, 19, 34393468.

30 V. Bellas and M. Rehahn, Angew. Chem., Int. Ed., 2007, 46, 5082-5104.

31 K. Kulbaba and I. Manners, Macromol. Rapid Commun., 2001, 22, 711-724.

32 F. He, T. Gadt, I. Manners and M. A. Winnik, J. Am. Chem. Soc., 2011, 133, 9095-9103.

33 G. A. Ozin and A. C. Arsenault, Mater. Today, 2008, 11, 44-51. 34 M. Mazurowski, M. Gallei, J. Li, H. Didzoleit, B. Stühn and M. Rehahn, Macromolecules, 2012, 45, 8970-8981.

35 J. Elbert, M. Gallei, C. Rüttiger, A. Brunsen, H. Didzoleit, B. Stühn and M. Rehahn, Organometallics, 2013, 32, 58735878.

36 J. Elbert, F. Krohm, C. Rüttiger, S. Kienle, H. Didzoleit, B. N. Balzer, T. Hugel, B. Stühn, M. Gallei and A. Brunsen, Adv. Funct. Mater., 2014, DOI: 10.1002/adfm.201302304.

37 J. Elbert, J. Mersini, N. Vilbrandt, C. Lederle, M. Kraska, M. Gallei, B. Stühn, H. Plenio and M. Rehahn, Macromolecules, 2013, 46, 4255-4267.

38 M. George and G. J. Hayes, Polymer, 1974, 15, 397-400.

39 M. George and G. J. Hayes, J. Polym. Sci., Polym. Chem. Ed., 1976, 14, 475-488.

40 C. G. Hardy, L. Ren, T. C. Tamboue and C. Tang, J. Polym. Sci., Part A: Polym. Chem., 2011, 49, 1409-1420.

41 C. G. Hardy, L. Ren, S. Ma and C. Tang, Chem. Commun., 2013, 49, 4373-4375. 